Research Paper

\title{
Totally Implantable Venous-Access Device Infection Causing Hematogenous Prosthetic Joint Infection: A Retrospective Case Series
}

\author{
Fernanda Medina ${ }^{1}$, Vanina Meyssonnier ${ }^{1,2}$, Valérie Zeller ${ }^{1,2}$, Beate Heym ${ }^{1,3}$, Jean-Marc Ziza ${ }^{1,2}$, Simon Marmor ${ }^{1,4} \bowtie$ \\ 1. Bone-and-Joint Infections Referral Center, Hôpital Croix Saint-Simon, 125, rue d'Avron 75020 Paris, France \\ 2. Internal Medicine Department, Hôpital Croix Saint-Simon, 125, rue d'Avron 75020 Paris, France \\ 3. Microbiology Department, Hôpital Croix Saint-Simon, 125, rue d'Avron 75020 Paris, France \\ 4. Orthopedic Surgery Department, Hôpital Croix Saint-Simon, 125, rue d'Avron 75020 Paris, France \\ $\triangle$ Corresponding author: Vanina Meyssonnier, MD-PhD, Bone-and-Joint Infections Referral Center, Groupe Hospitalier Diaconesses-Croix Saint-Simon, 125, \\ rue d'Avron 75020 Paris, France. Phone: +33 (0)1 43864172; Fax: +33 (0)1 43862309; e-mail: vmeyssonnier@hopital-dcss.org \\ (C) Ivyspring International Publisher. This is an open access article distributed under the terms of the Creative Commons Attribution (CC BY-NC) license \\ (https://creativecommons.org/licenses/by-nc/4.0/). See http://ivyspring.com/terms for full terms and conditions.
}

Received: 2018.03.05; Accepted: 2018.08.21; Published: 2018.10.31

\begin{abstract}
Introduction: Prosthetic joint infections (PJIs) can be acquired hematogenously from a distant site or device. Notably, $30 \%-40 \%$ of patients with PJls have Staphylococcus aureus bacteremia. No case reports or series of PJls acquired from totally implantable venous-access device (TIVAD) infection or colonization have been published. This study was undertaken to describe epidemiological, clinical, microbiological and radiological characteristics of such PJls, their treatments and outcomes.

Methods: This retrospective study included all patients, identified in a prospective French Bone-and-Joint Infections Referral Center cohort treated between 2004 and 2017, with PJI secondary to TIVAD infection, with the same microbiologically documented microorganism isolated from both.

Results: We describe six consecutive hematogenous PJls (4 women, 2 men; median age: 66.5 years) acquired from TIVAD primary infections. The main infection risk factors were malignancy $(n=5)$ and prior septic arthritis $(n=2)$. Four participants' TIVADs were implanted for chemotherapy, preceding the prosthesis for one patient. The median TIVAD-implantation-to-symptom-onset interval was 12 months. Microorganisms were Staphylococcus epidermidis $(n=4)$, Staphylococcus capitis $(n=1)$ and Staphylococcus aureus $(n=1)$. All TIVADs were removed. Five participants received curative treatment, with a median of 12 weeks of antibiotics. After median follow-up of 42 months, none have relapsed.
\end{abstract}

Conclusions: When PJI occurs in a patient with a TIVAD, the latter must be tested as a potential source of the prosthesis infection. Conversely, PJls must sought in all patients with bacteremia.

Key words: prosthetic joint infection; hematogenous dissemination; catheter infection.

\section{Introduction}

Prosthetic joint infections (PJIs) are rare, but severe, affections. The average infection rates are $\sim 0.5 \%$ and $\sim 1.5 \%$ for hip and knee prostheses, respectively [1, 2], with 2-year mortality exceeding $10 \%$ [3]. PJIs may be acquired by hematogenous dissemination. Venous devices can be responsible for bacteremia and contaminate any existing prosthetic joint.

Totally implantable venous-access devices (TIVADs) have become an indispensable tool for the management of patients needing long-term intravenous treatments [4]. Infection is the main complication of TIVAD use, with an incidence of $\sim 0.8$ per1000 catheter days, with risk of infection estimated 
to be between $5 \%$ and $10 \%$ [5]. To our knowledge, no case report on PJIs attributable to TIVAD infection or colonization has been published. The objective of this study was to describe the epidemiological and microbiological characteristics of PJIs secondary to TIVAD infection, their treatments and outcomes.

\section{Patients and Methods}

This retrospective study included all patients with PJI secondary to TIVAD infection, selected from a prospective cohort of patients treated in a French Bone-and-Joint Infections Referral Center between January 2004 and December 2017, and entered in a PJI registry. Patients were followed for 2 years with regular consultation at our Center.

The following criteria defined PJI secondary to TIVAD infection: 1) PJI occurring after a symptom-free interval of at least 1 month after prosthesis implantation, with sudden onset of signs, such as pain, joint dysfunction, febrile or not and/or chills. PJI was confirmed by isolation of the same microorganism species from at least two cultures of joint-fluid aspirates and/or intraoperative tissue and/or bone samples; 2) at least one positive culture of TIVAD and peripheral blood samples drawn simultaneously, when possible, and, TIVAD-blood culture bacterial growth detection 2 hours earlier than peripheral blood favoring infection of the former, or a positive TIVAD-blood culture even without bacteremia; 3) microbiological concordance: the same species with same resistance-phenotype profile is isolated from both devices; 4) chronological compatibility: the TIVAD was implanted before the PJI was diagnosed; and 5) TIVAD had to be confirmed as the unique source of secondary PJI.

\section{Microbiological methods [6]}

Blood culture bottles, one each containing either aerobic or anaerobic medium and labeled with patient's information, were inoculated under sterile conditions with blood samples drawn aseptically and transported immediately to the Microbiology Laboratory. The bottles were incubated in the automated BacT/ALERT® system (bioMérieux, Marcy l'Étoile, France) for 5 days. When the system detected bacterial growth, blood was subcultured on PolyViteX (PVX)-chocolate and Columbia agar plates (BioMérieux) that were then incubated under aerobic and anaerobic conditions for 48 hours.

Regarding the TIVAD, the port body and attached tunneled catheter were sent to the Microbiology Laboratory in a sterile recipient. Two milliliters of Schaedler broth were added to the device and vortexed. The resulting suspension was inoculated onto a PVX-chocolate plate and Columbia agar plate (bioMérieux) that were then incubated under aerobic and anaerobic conditions for 48 hours. The initial recipient containing the Schaedler broth was incubated at $37^{\circ} \mathrm{C}$ for 7 days and controlled daily for visible growth.

Bacteria were identified to species with the rapid ID 32A kit (bioMérieux) and, since 2012, by mass spectrometry (MALDI biotyper, Bruker Daltoni $\mathrm{GmbH}$, Breme, Germany). Antibiotic-susceptibility was determined with the agar-diffusion test, according to the French Society of Microbiology recommendations.

\section{Results}

Among 845 PJIs treated between 2004 and 2017, $292(34 \%)$ were secondary to a distant infection site. Six $(2 \%)$ patients, whose TIVAD infections were the sole PJI source, were included in the study (4 women, 2 men; median age \{range\}: $66.5\{51-78\}$ years). Their individual characteristics are summarized in Table 1.

Several risk factors for PJI could be identified in these patients, based on [7]: antiproliferative chemotherapy, either past $(n=4)$ (up to 3 years prior to the first PJI symptoms) or ongoing $(n=1)$; cancer $(n=5)$; prior PJI $(n=2)$. Two patients had had prior PJI: Patient 3 's with different microorganisms between the two PJIs: methicillin-resistant $S$. epidermidis for the first infection and Staphylococcus capitis for the second. The TIVAD had been implanted for administration of intravenous antibiotics but was never used. The treatment of the first PJI had been terminated for 6 months when the second was diagnosed. Patient 5's first PJI had occurred 2 years earlier and microbiological findings were unavailable; she had a TIVAD for chemotherapy.

The hip was infected in all patients and one had hip and knee PJIs.

TIVAD infection generated no local signs. The main symptoms were: joint pain $(n=6,100 \%)$, limb swelling $(n=1)$. Radiological signs were prosthesis loosening $(n=2,30 \%)$ and pseudarthrosis $(n=1)$.

For every case, PJI etiological investigations led to the diagnosis of TIVAD infection. Five never had any TIVAD-related symptoms and two developed signs of sepsis when the TIVAD was being used.

Patient 2's TIVAD blood culture became growth-positive $\geq 2$ hours before peripheral blood culture. The other microbiological results are detailed in Table 1.

All patients' cultures of the removed TIVADs, as described above, grew pathogens consistent with the infection. One patient was infected with two $S$. epidermidis strains, both of which were found on the TIVAD and the joint. TIVADs were removed 0-5 days before prosthesis removal for five patients; the other 
prosthesis had been removed 2 months earlier in another hospital. All six patients had normal trans-thoracic echocardiograms.

\section{None of the patients had persistent bacteremia}

Five patients received curative medical and surgical treatments (prosthetic exchange), with a duration of antibiotics from 6 to 14 weeks. The patient 3 's infected with two organisms, S. capitis and Candida albicans, received 12 weeks of antibacterial and 22 weeks of antifungal therapy. Every patient initially received intravenous antibiotics for a median of 14 days, followed by oral therapy. Rifampicin was given to five patients but switched to clindamycin for one because of digestive intolerance; it was not used for another because of feared interactions with other medications. Patient 5, unable to undergo surgery, received prolonged oral suppressive antibiotics. No TIVAD was reimplanted into five patients and information was unavailable for the sixth.

After median follow-up of 42 months [range: 5 months -7 years], no relapses have occurred. One patient was lost-to-follow-up.

\section{Discussion}

Herein, we described the clinical and microbiological characteristics of six PJIs secondary to TIVAD infections and their outcomes. They represented $2 \%$ of all hematogenous PJIs in our prospective cohort, treated in a Bone-and-Joint Infections Referral Center in France. To our knowledge, this is the first study on hematogenous PJIs caused by TIVAD infection. Hematogenous $S$. aureus seeding to prosthetic joints is a feared complication. Only one of these six patients was infected with $S$. aureus. S. epidermidis, the most frequently isolated bacterium, is a frequent pathogen causing TIVAD infections but only rarely associated with hematogenous spread [8]. It should be kept in mind, given the number of intravenous devices in patients with prosthetic joints, that the percentage of coagulase-negative staphylococci responsible for PJIs is likely low. Moreover, antibiotic-resistance levels herein were surprisingly low among these multi-hospitalized patients.

Table 1. Characteristics of the six patients with TIVAD-attributed PJls. Chemo: chemotherapy; AZA, azathioprine; MSSE: methicillin-susceptible Staphylococcus epidermidis; MSSA: methicillin-susceptible Staphylococcus aureus; TBC/PBC: TIVAD/peripheral blood culture; RH: right hip; LH: left hip; LK: left knee. SXT: sulfamethoxazole; FUS: fusidic acid; FOS: fosfomycin; ERY: erythromycin. NA: no available data.

\begin{tabular}{|c|c|c|c|c|c|c|c|c|c|c|c|c|}
\hline & & TIVAD & & & Prosthesis & & & PJI & & & & Antibiotic \\
\hline $\begin{array}{l}\text { Patient/ } \\
\text { sex/age }\end{array}$ & $\begin{array}{l}\text { Infection risk } \\
\text { factors }\end{array}$ & Reason & $\begin{array}{l}\text { Implantation } \\
\mathrm{yr}\end{array}$ & $\begin{array}{l}\text { Infection } \\
\text { yr }\end{array}$ & Reason & $\begin{array}{l}\text { Implantation } \\
\mathrm{yr}\end{array}$ & Joint & Year & $\begin{array}{l}\text { Symptom } \\
\text { onset } \\
\text { post-TIVAD } \\
\text { (mo) }\end{array}$ & $\begin{array}{l}\text { Pathogen(s) } \\
\text { (sites) } \\
\text { [resistance] }\end{array}$ & $\begin{array}{l}\text { Surgical } \\
\text { treatment }\end{array}$ & $\begin{array}{l}\text { duration } \\
\text { (wk) }\end{array}$ \\
\hline $1 / F / 72$ & $\begin{array}{l}\text { Aza, cancer, } \\
\text { obesity, } \\
\text { chemo }\end{array}$ & Chemo & 2002 & 2004 & Arthrosis & 1993 & $\mathrm{LH}$ & 2004 & 24 & $\begin{array}{l}\text { MSSE (PBC, } \\
\text { TIVAD, joint } \\
\text { fluid); [STX] } \\
\text { both sites }\end{array}$ & 2 stage & 6 \\
\hline $2 / F / 66$ & $\begin{array}{l}\text { Cancer, } \\
\text { chemo }\end{array}$ & Chemo & 2003 & 2005 & Arthrosis & 2004 & $\mathrm{LH}$ & 2005 & 15 & $\begin{array}{l}\text { MSSE (PBC, } \\
\text { TBC, TIVAD, } \\
\text { intra-operative } \\
\text { hip samples) }\end{array}$ & 1 stage & 12 \\
\hline $3 / \mathrm{M} / 67$ & $\begin{array}{l}\text { Prior PJI } \\
\text { (S.epidermidis) }\end{array}$ & Antibiotics & 2006 & 2009 & Arthrosis & 2006 & $\mathrm{RH}$ & 2009 & 12 & $\begin{array}{l}\text { S. capitis } \\
\text { (TIVAD, } \\
\text { intra-operative } \\
\text { samples), [FUS, } \\
\text { FOS] } \\
\text { prosthesis } \\
\text { only; C. albicans } \\
\text { (PBC, } \\
\text { intra-operative } \\
\text { samples) }\end{array}$ & 2 stage & 14 \\
\hline 4/F/78 & $\begin{array}{l}\text { Cancer, } \\
\text { chemo }\end{array}$ & $\begin{array}{l}\text { Parenteral } \\
\text { nutrition }\end{array}$ & 2009 & 2010 & Arthrosis & $\begin{array}{l}2004 \text { (RH), } \\
2005 \text { (LK) }\end{array}$ & $\begin{array}{l}\text { RH } \\
+\mathrm{LK}\end{array}$ & 2010 & 12 & $\begin{array}{l}\text { MSSE (TBC, } \\
\text { TIVAD, } \\
\text { intra-operative } \\
\text { samples); } \\
\text { [ERY] both } \\
\text { sites }\end{array}$ & 1 stage & 13 \\
\hline $5 / F / 51$ & $\begin{array}{l}\text { Prior PJI, } \\
\text { cancer, chemo }\end{array}$ & Chemo & 2010 & 2010 & Arthrosis & 2009 & $\mathrm{LH}$ & 2011 & 1 & $\begin{array}{l}\text { MSSA (TIVAD, } \\
\text { intra-operative } \\
\text { samples) }\end{array}$ & NA & Suppressive \\
\hline $6 / \mathrm{M} / 60$ & $\begin{array}{l}\text { Cancer, } \\
\text { chemo }\end{array}$ & Chemo & 2004 & 2007 & Osteo-necrosis & 1993 & RH & 2007 & $\begin{array}{l}\text { Preceded } \\
\text { TIVAD }\end{array}$ & $\begin{array}{l}\text { MSSE (TIVAD, } \\
\text { intra-operative } \\
\text { samples) }\end{array}$ & 2 stage & 6 \\
\hline
\end{tabular}


This study was mainly limited by the rarity of PJIs secondary to TIVAD infection and the absence of TIVAD-infection symptoms, which may be explained by the low virulence of coagulase-negative staphylococci. It may be discussed that because of the low-grade infections of coagulase-negative staphylococci producing little or no symptoms, they may have enough time to grow until they have the capacity for hematogenous seeding on another foreign material in the body. In contrast, $S$. aureus may produce rapidly local clinical signs of TIVAD infection leading to removal of the TIVAD before seeding on to prosthesis.

Interpretation was made more difficult by the different resistance phenotypes like for of patient 3's who had different TIVAD and PJI infecting strains of S.capitis; moreover we found candidemia in peripheral blood but TIVAD-blood cultures were not obtained. The same Candida strain was found on the prosthetic joint, but not the TIVAD culture. Even if all the criteria are not present to confirm the TIVAD as source if PJI, doubt must benefit the patient and TIVAD must be removed. For patient 6, symptom chronology could lead us to think that the PJI was responsible for hematogenous seeding of the TIVAD, but the same strain was isolated from both devices and it was impossible to determine the primary infection site. Notably, exact identity between TIVAD and prosthesis strains was not confirmed by molecular biology. When no growth-positive peripheral blood cultures are available, it can be imagined that a common colonizing microorganism might have infected both devices, without hematogenous seeding and for the same reason as above, TIVAD must be removed.

When a TIVAD is present, its possible infection must be sought as a PJI source. Indeed, for secondary PJIs, their origins must be treated before surgery or before the end of antimicrobial therapy. TIVADs were removed from all six patients before surgical treatment of the PJI for five. Pertinently, TIVADs should be removed as soon as they are no longer needed. Conversely, every prosthetic joint should be verified when a TIVAD infection occurs, particularly with bacteremia. The actual risk of secondary PJI in a context of bacteremia remains to be determined in future studies.

\section{Abbreviations}

PJI: prosthetic joint infection; TIVAD: totally implantable venous-access device.

\section{Ethics Approval}

Not applicable.

\section{Competing Interests}

The authors have declared that no competing interest exists.

\section{References}

1. Huotari K, Peltola M, Jämsen E. The incidence of late prosthetic joint infections. Acta Orthop 2015; 86: 321-5.

2. Lamagni T. Epidemiology and burden of prosthetic joint infections. J Antimicrobial Chemother 2014; 69 (Suppl 1): 5-10.

3. Zmistowski B, Karam JA, Durinka JB, Casper DS, Parvizi J. Periprosthetic joint infection increases the risk of one-year mortality. J Bone Joint Surg Am 2013; 95: 2177-84.

4. Journées Nationales d'Infectiologie. Jean-Christophe Lucet. Pose, prévention et surveillance des chambres à cathéters implantable. 2012. http://www.infectiologie.com/UserFiles/File/medias/JNI/JNI12/2012-JNICIP-lucet.pdf

5. Groupe de Travail CClin Sud-Ouest. Recommandations pour la réduction du risque infectieux lié aux chambres à cathéter implantables. 2001. Available at: http://www.cclin-arlin.fr/nosopdf/doc01/0009867.pdf

6. Comité de l'Antibiogramme de la Société Française de Microbiologie (CASFM), European Committee on antimicrobial susceptibility testing of the European Society of Clinical Microbiology and Infectious Diseases (EUCAST). Recommendations 2014 available at: http://www.sfm-microbiologie.org/ UserFiles/files/casfm/CASFMV2_030915.pdf

7. Tande AJ, Patel R. Prosthetic joint infection. Clin Microbiol Rev [Internet]. 2014 Apr [cited 2018 Jul 10];27(2):302-45. Available at: https://www.ncbi.nlm.nih.gov/pmc/articles/PMC3993098/

8. Lebeaux D, Larroque B, Gellen-Dautremer J, Leflon-Guibout V, Dreyer C, Bialek S, et al. Clinical outcome after a totally implantable venous access port-related infection in cancer patients: a prospective study and review of the literature. Medicine [Internet]. 2012 Nov [cited 2018 Jul 12];91(6):309. Available from: https://journals.lww.com/md-journal/fulltext/2012/11000/Clinical

Outcome_After_a_Totally_Implantable.2.aspx 